\title{
Regression of White Matter MRI Abnormalities in Nonvasculitic Autoimmune Inflammatory Meningoencephalitis following Intravenous Immunoglobulin
}

\author{
Daniele Imperiale ${ }^{a}$ Giulia Guastamacchia ${ }^{a}$ Sergio Duca ${ }^{c}$ Lucia Appendino ${ }^{a}$ \\ Giorgio Marietti $^{b}$ Alessandra Romito ${ }^{b}$ Cristiana Atzori $^{a}$ Carlo Buffa ${ }^{a}$ \\ ${ }^{a}$ Dipartimento Neurologia e Centro Prioni DOMP, e ${ }^{b}$ Dipartimento Diagnostica di Laboratorio, \\ Ospedale Maria Vittoria, e 'Servizio RMN, Ospedale Koelliker, Torino, Italia
}

\begin{abstract}
Dear Sir,
Nonvasculitic autoimmune inflammatory meningoencephalitis (NAIM) is a rare cause of usually steroid-responsive encephalopathy characterized by multiple and nonspecific autoimmune serologic associations [1]. Hashimoto encephalopathy may represent an acute or subacute form of NAIM associated with high titers of antithyroid antibodies independent of thyroid function [2]. The clinical picture is variable and usually involves delirium, hallucinations, myoclonus, seizures and strokelike episodes [3]. In steroid-resistant patients other treatments such as plasma exchange and intravenous immunoglobulin (IVIG) have been employed [4, 5]. MRI studies may provide evidence of focal or diffuse, nonenhancing altered signal areas $[2,3]$. Normalization of NAIM-associated white matter MRI alterations has been reported following steroid therapy but not following IVIG and plasma exchange [3, $6,7]$.
\end{abstract}

\section{Case Report}

In December 2004 a 33-year-old woman was admitted to our emergency room due to acute onset of confusion, agitation and hallucinations. Cranial CT and brain MRI were normal. EEG was diffusely slow with rare bilateral spike and wave dis- charges. The CSF was proteinaceous $(2.42$ $\mathrm{g} / \mathrm{l})$ but acellular and sterile. On suspicion of viral encephalitis, the patient received treatment with both systemic acyclovir (dose/day) and methylprednisolone (dose/ day), resulting in rapid improvement of mental status. After 10 days acyclovir was stopped and methylprednisolone was tapered off over 5 days.

Four weeks later (January 2005) the patient was referred to our neurological ward due to persistent confusion and two generalized tonic-clonic seizures. A new CSF examination disclosed raised protein levels $(0.92 \mathrm{~g} / \mathrm{l})$ without cells. EEG was diffusely slow. Neuroimaging studies were unremarkable. Serum titers of anti-thyroid antibodies were elevated (anti-thyroperoxidase Ab: $591 \mathrm{U} / \mathrm{ml}$, anti-thyroglobulin Ab: $905 \mathrm{U} / \mathrm{ml}$ ), whereas thyroid-stimulating hormone, free triiodothyronine and free thyroxine were in the normal range. A presumptive diagnosis of NAIM associated with thyroid autoimmunity was made. Systemic methylprednisolone and valproic acid were administered. The clinical picture promptly improved and she was discharged after 10 days on oral valproic acid 1,000 mg/day.

The patient was admitted again 3 weeks later (February 2005) due to drows- iness, confusion and tremulousness. In spite of high-dose systemic methylprednisolone (1,000 mg/day for 1 week), only a mild improvement in mental status was observed. Within a few days the patient developed a dissociative state with delusions, diffuse paratonia, upper limb tremor and dystonia. A brain MRI study revealed bilaterally high $\mathrm{T}_{2}$-weighted and FLAIR signals involving the hemispheric deep white matter with no contrast enhancement (fig. 1A). EEG showed diffuse bilaterally slow activity in the theta-delta range. CSF examination was unremarkable. Tau concentration in CSF was normal and 14-3-3 protein was not detected. Thyroid function was also normal. Serum anti-thyroperoxidase antibody titers were raised (166 U/ml). IVIG ( $2 \mathrm{~g} / \mathrm{kg}$ in 5 days) was administered, leading to dramatic clinical status improvement within $24 \mathrm{~h}$. The EEG pattern also returned to normal within a few days. The patient was discharged completely recovered. Brain MRI was repeated 1 month later (April 2006), highlighting an almost complete regression of white matter abnormalities (fig. 1B). The last follow-up visit, in April 2006, saw the patient therapy-free with no report of other relapses.

\section{KARGER}

Fax +4161306 1234 E-Mail karger@karger.ch www.karger.com
(C) 2007 S. Karger AG, Basel 0014-3022/07/0574-0244\$23.50/0

Accessible online at: www.karger.com/ene
Dr. Daniele Imperiale

Dipartimento Neurologia e Centro Prioni DOMP

ASL 3 Ospedale Maria Vittoria, Via Cibrario 72

IT-10144 Torino (Italy)

Tel. +39 011439 3296, Fax +39011439 3503, E-Mail imperiale@asl3.to.it 
Fig. 1. MRI coronal slices, FLAIR sequences. A Bilateral white matter hyperintensity consistent with severe leukoencephalopathy. B Almost complete regression of white matter abnormalities after IVIG administration (1 month later).

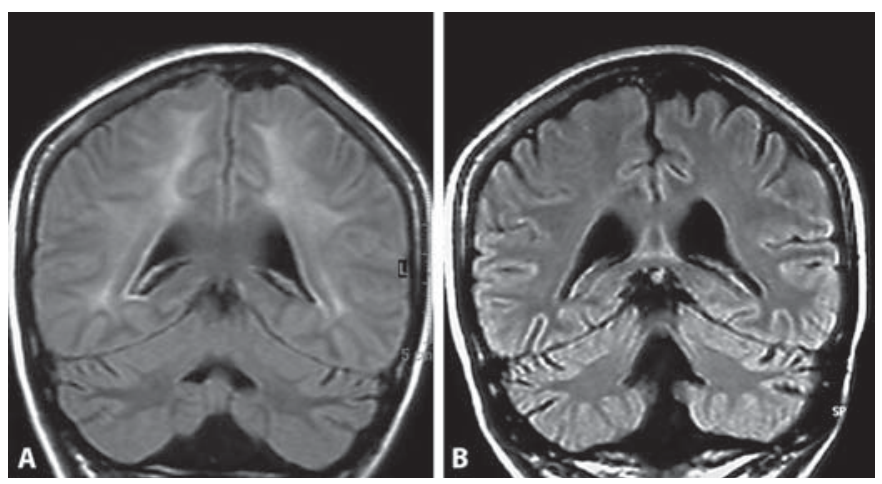

\section{Discussion}

NAIM is a clinically heterogeneous encephalopathy with multiple and nonspecific autoimmune associations [1]. When associated with thyroid autoimmunity, it is also referred to as Hashimoto's encephalopathy or steroid-responsive encephalopathy with autoimmune thyroiditis [2,3]. Its clinical and imaging features have been attributed to an autoimmune meningoencephalitic process, but pathologic data are scarce due to usually excellent steroid responsiveness. A modest perivascular lymphocytic infiltration throughout the brain (in particular the medulla) and the leptomeninges has been demonstrated in a few cases [8-10]. Such a mild histologic substrate presumably explains the lack of MRI abnormalities in most NAIM patients. In any case, nonenhancing lesions on $\mathrm{T}_{2^{-}}$ weighted scans are present in $25-50 \%$ of cases $[2,3]$. Diffuse white matter abnormalities on MRI scans are described in NAIM with thyroid autoimmunity $[2,3,6]$. Myelin loss with perivascular lymphocytic infiltrates and relative axonal sparing was recently described in a case of NAIM that had undergone brain biopsy [11]. Thus, white matter MRI abnormalities may be related to a primary demyelinating process concomitant with autoimmune thyroiditis. In the present paper we reported a steroid-resistant NAIM patient with a severe, diffuse leukoencephalopathy that resolved after IVIG administration. Reversible white matter MRI abnormalities have been described following corticosteroid treatment $[6,7]$. To our knowledge, the IVIG regression of both clinical and MRI signs in NAIM with thyroid autoimmunity has not been previously reported. Indeed, steroid-resistant NAIM cases responding to IVIG have been described in the literature, but MRI data are lacking [2, 5]. IVIG is an established treatment for immune-mediated demyelinating neuropathy and, as a result of its immunomodulatory and presumptive remyelinating properties, is also regarded as a second-line therapy of multiple sclerosis [12]. The complete clinical recovery and the almost complete normalization of the MRI picture in the present case further support IVIG as a valuable and long-term effective therapy in steroid-resistant NAIM patients.

\section{Acknowledgment}

The 'Centro DOMP' is supported by 'Regione Piemonte Ricerca Sanitaria Finalizzata 2002-2003-2004'.

\section{References}

1 Caselli RJ, Boeve BF, Scheithauer BW, O’Duffy JD, Hunder GG: Nonvasculitic autoimmune inflammatory meningoencephalitis (NAIM): a reversible form of encephalopathy. Neurology 1999;53:1579.

2 Chong JY, Rowland LP, Utiger RD: Hashimoto encephalopathy: syndrome or myth? Arch Neurol 2003;60:164-171.

3 Castillo P, Woodruff B, Caselli R, Vernino S, Lucchinetti C, Swanson J, Noseworthy J, Aksamit A, Carter J, Sirven J, Hunder G, Fatourechi V, Mokri B, Drubach D, Pittock S, Lennon V, Boeve B: Steroid-responsive encephalopathy associated with autoimmune thyroiditis. Arch Neurol 2006;63:197-202.
4 Boers PM, Colebatch JG: Hashimoto's encephalopathy responding to plasmapheresis. J Neurol Neurosurg Psychiatry 2002;73:601.

5 Jacob S, Rajabally A: Hashimoto's encephalopathy: steroid resistance and response to intravenous immunoglobulins. J Neurol Neurosurg Psychiatry 2005;76:455-456.

6 Bohnen NIL, Parnell KJ, Harper CM: Reversible MRI findings in a patient with Hashimoto's encephalopathy. Neurology 1997;49:246-247.

7 Pozo-Rosich P, Pablo Villoslada P, Canton A, Simo R, Rovira A, Montalban X: Reversible white matter alterations in encephalopathy associated with autoimmune thyroid disease. J Neurol 2002;242:1063-1065.

8 Nolte KW, Unbehaun A, Sieker H, Kloss TM, Paulus W: Hashimoto encephalopathy: a brainstem vasculitis? Neurology 2000;54: 769-770.

9 Duffey P, Yee S, Reid IN, Bridges LR: Hashimoto's encephalopathy: postmortem findings after fatal status epilepticus. Neurology 2003;61:1124-1126.

10 Josephs KA, Rubino FA, Dickson D: Nonvasculitic autoimmune inflammatory meningoencephalitis. Neuropathology 2004;24: 149-152.

11 Mahad DJ, Staugaitis S, Ruggieri P, Parisi J, Kleinschmidt-Demasters BK, Lassmann $\mathrm{H}$, Ransohoff RM: Steroid-responsive encephalopathy associated with autoimmune thyroiditis and primary CNS demyelination. J Neurol Sci 2005;228:3-5.

12 Jorgensen SH, Sorensen PS: Intravenous immunoglobulin treatment of multiple sclerosis and its animal model, experimental autoimmune encephalomyelitis. J Neurol Sci 2005;233:61-65. 